\title{
EVALUACIÓN APROXIMADA DE LA ACELERACIÓN ABSOLUTA PARA UNA FORMA MODAL EN SISTEMAS DE MÚLTIPLES GRADOS DE LIBERTAD
}

\author{
Juan Diego Jaramillo ${ }^{(1)}$
}

\begin{abstract}
RESUMEN
Se presenta una propuesta para la estimación de la aceleración absoluta máxima en los diferentes niveles de un sistema de múltiples grados de libertad en función de la forma modal y del espectro de respuesta de aceleraciones, que tradicionalmente se propone en los reglamentos. La propuesta está basada en la combinación de una respuesta modal con la señal de excitación cuando estas se consideran armónicas. El error promedio de la propuesta presentada cuando se compara con la solución exacta calculada usando series de tiempo es de aproximadamente $7 \%$, que contrasta con errores promedio del orden de $20 \%$ y mayores en otras tres propuestas presentadas en códigos de diseño sísmico actualmente vigentes.
\end{abstract}

\section{SUMMARY}

A proposal is presented for estimating the maximum absolute acceleration at different levels in a multi-degree of freedom systems. This is done in terms of the modal shape and the acceleration response spectrum, which traditionally is part of seismic regulations. The proposal is based on the combination of a modal response with the excitement signal when they both are considered as harmonics. The present proposal has an $\sim 7 \%$ average error when it is compared with exact solutions calculated using time series. This error contrasts with $20 \%$ and greater average errors found in other three analyzed proposals presented in three seismic codes.

\section{INTRODUCCIÓN}

En el diseño de los elementos estructurales de un sistema sometido a aceleraciones en la base se parte de la historia de los desplazamientos relativos respecto a la base, que son justamente la solución de la ecuación dinámica (ec. 1). En algunos casos, esta solución se presenta en la forma de fuerzas estáticas equivalentes que reproducen los desplazamientos en un instante determinado o en el instante del desplazamiento máximo, cuando el sistema se idealiza como de un grado de libertad con una forma modal definida. Es claro, entonces, que los esfuerzos internos, de quienes depende directamente el diseño adoptado, están en función de los desplazamientos relativos de la estructura respecto a la base, lo que justifica plenamente la manera como se describe la acción

\footnotetext{
Artículo recibido el 2 de abril de 2003 y aprobado para su publicación el 19 de septiembre de 2003. Se aceptarán comentarios y/o discusiones hasta cinco meses después de su publicación.

${ }^{(1)}$ Departamento de Ingeniería Civil, Universidad EAFIT, A.A. 3300, Medellín, Colombia, ijarami@eafit.edu.co
} 
sísmica en la mayoría de los códigos sismo-resistentes alrededor del mundo, a través de un espectro de respuesta.

En la mayoría de estos códigos sismo resistentes el espectro de respuesta propuesto corresponde al espectro de seudoaceleraciones, que está relacionado directamente con las fuerzas estáticas que reproducen el desplazamiento máximo relativo de un sistema de un grado de libertad. Esta representación de la acción sísmica tiene que ver más con un esquema de pensamiento, en el que las solicitaciones estructurales se presentan como fuerzas y no como desplazamientos, que con un beneficio claro en los procedimientos y resultados de diseño. En algunos reglamentos más modernos se especifica directamente el espectro de respuesta de desplazamientos, esta vez junto con un procedimiento de diseño basado en los desplazamientos relativos de la estructura respecto a la base.

Se quiere llamar la atención en este artículo, no al procedimiento de diseño de los elementos estructurales sino al de los elementos no estructurales, particularmente aquellos adheridos a la estructura en un solo punto y de alta rigidez, que interactúan en bajo grado con la estructura, y que justamente por razón de no aportar ni a la resistencia ni a la rigidez del sistema, solo a la masa, es práctica común no involucrarlos en el modelo. Ahora, el hecho de que no aporten ni a la resistencia ni a la rigidez del sistema no significa que no estén sometidos a solicitaciones que necesariamente hay que estimar para evaluar la propia integridad de estos elementos y la de los sistemas que los sujetan a la estructura.

Las preguntas a responder son: ¿A que historia de aceleraciones absolutas está sujeto un elemento no estructural anclado en alguna parte de la estructura? A esta pregunta se responde fácilmente puesto que se supone que la acción sísmica está definida por un acelerograma y la aceleración absoluta que se está buscando se calcula como la suma de la historia de las aceleraciones relativas al suelo, solución de la ecuación dinámica del sistema, y la historia de la aceleración del sismo en la base de la estructura. La segunda pregunta que se haría y que es la más común es: ¿A que aceleración absoluta máxima está sujeta un elemento no estructural anclado en alguna parte de la estructura? A esta pregunta no se responde tan fácilmente puesto que en este caso se supone que la acción sísmica está representada por un espectro de respuesta, que representa justamente la respuesta de la estructura en términos de desplazamientos relativos o de seudoaceleraciones, y solo se tiene información parcial de la historia de las aceleraciones en la base de la estructura y de la historia de las aceleraciones absolutas en un sitio particular de la estructura.

A continuación se explora el tema con detalle y se propone un procedimiento aproximado para responder a la segunda pregunta planteada con un error aceptable para la mayoría de las aplicaciones.

Especialmente para diseño de elementos secundarios en plantas nucleares se han desarrollado procedimientos elaborados basados en su mayoría en la teoría de vibraciones aleatorias para estimar el llamado espectro de respuesta de piso, que de la misma manera que el espectro de respuesta para una estructura, define las características de la respuesta de osciladores de diferentes frecuencias y amortiguamientos, adosados a los diferentes niveles de la estructura 
principal cuando ésta es sometida a un movimiento en la base caracterizado por un espectro de respuesta. Ejemplos de estos procedimientos son los que presentan Singh (1980), Singh y Sharma (1985), e Igusa y Der Kiureghian (1985).

Es claro que una respuesta a la pregunta planteada en un párrafo anterior está dada como la ordenada del espectro de respuesta de piso correspondiente a un periodo nulo. La propuesta que aquí se plantea está orientada a presentar un planteamiento simple basado en el espectro de respuesta de seudoaceleraciones, que son los que ordinariamente se presentan en los reglamentos de diseño sísmico alrededor del mundo, sin tener que hacer uso de procedimientos más elaborados planteados en la teoría de vibraciones aleatorias, que están basados en espectros de velocidades o aceleraciones relativas como en el caso de las referencias citadas.

\section{PLANTEAMIENTO DEL PROBLEMA}

Se plantea a continuación el problema suponiendo que se conoce la historia de aceleraciones en la base de la estructura.

La ecuación dinámica de un sistema de múltiples grados de libertad se escribe:

$[\mathrm{M}] \underline{x}+[\mathrm{C}] \underline{\dot{x}}+[\mathrm{K}] \underline{x}=-[\mathrm{M}] a_{g}(t)$

donde, $[\mathrm{M}],[\mathrm{C}] \mathrm{y}[\mathrm{K}]$ representan las matrices de masa, amortiguamiento y rigidez, respectivamente, $\underline{x}, \underline{\dot{x}}$ y $\underline{x}$ representan los vectores de aceleración, velocidad y desplazamiento relativos, respectivamente, $\underline{1}$ representa el vector geométrico para asignación de masas y $a_{g}(t)$ representa la historia de aceleraciones en la base de la estructura.

Si $[\Theta]$ representa la matriz de formas modales, $\phi \dot{\phi}$, y $\underline{z}$ el vector de desplazamientos modales, $z^{j}$, soluciones de las ecuaciones dinámicas desacopladas,

$z^{j}+2 \xi^{j} w^{j} \dot{z}^{j}+w^{j^{2}} z^{j}=-a_{g}(t)$

donde $\xi^{j} \mathrm{y} w^{j}$ representan la relación de amortiguamiento y frecuencia circular propios del modo $j$, respectivamente, la historia de aceleraciones absolutas del grado de libertad $i$ debido al modo $j$, $a_{i}^{j}(t)$, se escribe como:

$a_{i}^{j}(t)=F P^{j} \phi_{i}^{j} z^{j}(t)+a_{g}(t)$

donde $F P^{j}$ es el factor de participación del modo $j$, que se escribe como:

$$
F P^{j}=\frac{\phi^{j^{T}}[\mathrm{M}] \underline{\underline{1}}}{\underline{\phi}^{j^{T}}[\mathrm{M}] \underline{\phi}^{j}}
$$


De manera sintética, la aceleración absoluta de algún grado de libertad y debida a alguna forma modal se puede escribir como:

$a_{i}^{j}(t)=\varphi_{i}^{j} z^{j}(t)+a_{g}(t)$

donde

$\varphi_{i}^{j}=F P^{j} \phi_{i}^{j}$

es un factor que depende de las formas modales y la matriz de masas.

La aceleración absoluta del grado de libertad $i$ y debida a la combinación de $n$ modos se escribe como:

$a_{i}(t)=\sum_{j=1}^{j=n}\left[\varphi_{i}^{j} z^{j}(t)\right]+a_{g}(t)$

En lo que sigue se hará referencia exclusivamente a una propuesta para evaluar la aceleración absoluta debida solo a un modo (ec. 5).

Lo interesante de la ec. 5 es que expresa que la aceleración absoluta de algún grado de libertad y debida a alguno de los modos de vibración se expresa como la combinación lineal de dos series de tiempo: $z^{j}(t)$ y $a_{g}(t)$.

Si la acción sísmica se especifica por medio de la aceleración en la base de la estructura, $a_{g}(t)$, entonces, $z^{j}(t)$ es fácilmente calculable resolviendo la ec. (2) por cualquiera de los métodos tradicionales, y la aceleración absoluta $a_{i}^{j}(t)$ es simplemente la combinación lineal de dos series de tiempo conocidas (ec. 5).

El problema se presenta cuando, como es usual en la mayoría de los códigos sismo resistentes alrededor del mundo, la acción sísmica se especifica a través de un espectro de diseño, que dependiendo de que tan juiciosamente haya sido especificado, se puede asimilar a una especie de envolvente de los espectros de respuesta de posibles sismos de diseño en el sitio. Esta descripción de espectro de diseño debe considerarse únicamente como ilustrativa porque ni es claro el procedimiento descrito para calcularlo, ni la definición de sismo de diseño. En lo que sigue se considerará, entonces, el espectro de diseño como el espectro de respuesta del "sismo de diseño" $a_{g}(t)$ para una determinada relación de amortiguamiento $\xi$.

En el caso anterior solo se conoce el valor máximo de la aceleración en la base, $a_{\max }$, definida como el corte del espectro de seudoaceleraciones con el eje de las ordenadas cuando en el eje de las abscisas se definen, como es usual, los periodos estructurales, y la seudoaceleración asociada a la frecuencia circular de vibración del modo $j, S a\left(w^{j}\right)$. Este último valor puede considerarse como una muy buena aproximación, con desviaciones menores al 1\%, de la 
aceleración absoluta de un sistema de un grado de libertad de amortiguamiento $\xi$ y frecuencia circular de vibración $w^{j}$ sujeto a un aceleración en la base igual a $a_{g}(t)$.

En otras palabras, no están completamente definidas ni $z^{j}(t)$ ni $a_{g}(t)$ pero está definido el valor máximo de $a_{g}(t), a_{\max }$, que es igual al valor máximo de $a_{i}^{j}(t)$ cuando $\varphi_{i}^{j}$ es igual a $0.0 \mathrm{y}$ está definido el valor máximo de $a_{i}^{j}(t)$ cuando $\varphi_{i}^{j}$ es igual a 1.0 , que es igual a $S a\left(w^{j}\right)$. La pregunta en este caso sería, ¿cuál es el valor máximo de $a_{i}^{j}(t), a_{i \max }^{j}$, para valores de $\varphi_{i}^{j}$ diferentes de 0.0 y 1.0 ?

\section{ANTECEDENTES}

Paulay y Priestley (1992) proponen, para efectos de estimar las aceleraciones a que estarían sometidos muros de mampostería ubicados a diferentes niveles, la siguiente aproximación:

$$
\begin{array}{ll}
a_{i_{\text {max }}^{j}}^{j}=a_{\text {max }}+\frac{S a-a_{\text {max }}}{H_{e q}} y & \left(0<\mathrm{y}<H_{e q}\right) \\
a_{i_{\text {max }}^{j}}^{j}=\frac{S a}{H_{e q}} y & \left(\mathrm{y}>H_{e q}\right)
\end{array}
$$

que significa que la aceleración absoluta máxima, $a_{i \max }^{j}$, es una función lineal de la altura desde la superficie del suelo, $y$, de tal manera que es igual a $a_{\max }$ a nivel de la superficie del suelo e igual a $S a$ a una altura igual a $H_{e q}$, altura en la que $\varphi_{i}^{j}$ es igual a 1.0 (ec. 6). A partir de esta altura, la aceleración absoluta máxima es proporcional a la altura $y$.

Más adelante se muestra como esta aproximación lineal, incluso para el primer modo de vibración de edificaciones homogéneas en altura, predice valores con desviaciones considerables respecto a los valores máximos de $a_{i}^{j}(t)$ estimados usando series de tiempo.

El título A de las Normas Colombianas para Diseño y Construcción Sismo Resistente, NSR-98, especifica, ec. A.2.9, que la aceleración en el punto de soporte de un elemento no estructural, $a_{i \max }^{j}$, se calcula como:

$$
\begin{aligned}
& a_{i \text { max }}^{j}=\frac{C_{v i} V_{s}}{m_{i} g} \leq 2 S a, \quad a_{i \text { max }}^{j} \geq \frac{A_{a} I}{2} \\
& C_{v i}=\frac{m_{i} y_{i}^{k}}{\sum_{i=1}^{n} m_{i} y_{i}^{k}}
\end{aligned}
$$


$V_{s}=S a \sum_{i=1}^{n} m_{i}$

donde $V_{s}$ es el cortante sísmico en la base del edificio, $C_{v i} V_{s}$ es la fuerza lateral aplicada al piso $i$ donde está anclado el elemento no estructural, y $m_{i}$ y $y_{i}$ son la masa de este piso y su altura desde el nivel de desplante, respectivamente. $n$ es el número de pisos del edificio, $g$ es la aceleración de la gravedad y $A_{a} I$ es la aceleración máxima a nivel del suelo. Esta propuesta está basada en el hecho de que la aceleración absoluta se puede aproximar al cociente entre las fuerzas estáticas equivalentes y la masa (por ejemplo Clough y Penzien, 1993), la cual resulta una muy buena aproximación siempre que el factor $\varphi_{i}^{j}$ sea igual a 1.0, en cuyo caso, como ya se mencionó, la aceleración absoluta es aproximadamente igual al espectro de seudoaceleraciones.

Ahora, dado que $C_{v i}$ es proporcional a la masa del piso y a la altura de éste desde el nivel de desplante de la estructura, $y_{i}$, elevado a la potencia $k$, función del periodo fundamental de la construcción, se concluye que en estas expresiones se considera que $\phi \dot{\phi}$ es proporcional a la altura desde el nivel de desplante elevado a la potencia $k, y^{k}$.

Para el nivel cero, es decir en el nivel de desplante, la aceleración absoluta estimada por la ec. 10 resulta igual a la mitad de la aceleración máxima del suelo y esto debido a la restricción de esta ecuación. Este hecho de por si ya involucra un error del 50\% ya que la aceleración absoluta máxima a nivel de desplante es igual a la aceleración máxima del suelo.

El otro punto de control de la aceleración absoluta es aquel en el que el factor $\varphi_{i}^{j}$ es igual a 1.0. En este nivel la aceleración absoluta debe ser igual a $S a$.

Con base en las ecs. 10, 11 y 12 y para el caso de un edificio con la masa uniformemente distribuida en la altura, se concluye que la aceleración a la altura $y$, en proporción a la altura total de la edificación, $H$, está dada por:

$a_{i \max }^{j}=S a(k+1)\left(\frac{y}{H}\right)^{k}$

Considerando que $\Phi$ es proporcional a la altura desde el nivel de desplante elevado a la potencia $k, y^{k}$, y haciendo uso de las ecs. 4 y 6 , resulta que la altura $H_{e q}$ en proporción a la altura total de la edificación, $H$, para la que $\varphi_{i}^{j}$ es igual a 1.0 está dada por:

$$
\frac{H_{e q}}{H}=\left(\frac{k+1}{2 k+1}\right)^{1 / k}
$$

Reemplazando en la ec. 13 la altura dada por la ec. 14, la aceleración absoluta para este nivel resulta igual a: 
$\left.a_{i \max }^{j}\right|_{H_{e q}}=\frac{(k+1)^{2}}{(2 k+1)} S_{a}$

Para cuatro valores representativos de $k$ se tienen los siguientes resultados:

$\begin{array}{lll}k=1.00 & H_{e q}=0.667 H & \left.a_{i \max }^{j}\right|_{H_{e q}}=1.333 S_{a} \\ k=1.25 & H_{e q}=0.702 H & \left.a_{i \max }^{j}\right|_{H_{e q}}=1.446 S_{a} \\ k=1.50 & H_{e q}=0.731 H & \left.a_{i \max }^{j}\right|_{H_{e q}}=1.563 S_{a} \\ k=2.00 & H_{e q}=0.775 H & a_{\left.i_{\text {max }}\right|_{H_{e q}}=1.800 S_{a}}\end{array}$

que muestran que a este nivel los errores cometidos en la estimación de $a_{i \max }^{j}$ van desde $33 \%$ para el caso $k=1.0$ hasta el $80 \%$ para el caso $k=2.0$.

Esta sobrestimación en la aceleración absoluta a nivel de la altura $H_{e q}$ es producto de la sobreestimación en la cortante basal, $V_{s}$, para un modo de vibración. Considerando formas modales proporcionales a $y^{k}$ es posible mostrar que la cortante basal debida a un solo modo y en estructuras de masa uniformemente distribuida en la altura se escribe como:

$V_{s}=S a \cdot M \cdot \frac{(2 k+1)}{(k+1)^{2}}$

donde $M$ es la masa total de la estructura.

La ec. 16 indica que la cortante basal asociada a modos como los que se están considerando es igual a la masa total de la estructura, $M$, multiplicada por la aceleración espectral, $\mathrm{Sa}$, esto es, la propuesta hecha por la norma NSR-98 (ec. 12), pero multiplicada por un factor que es justamente el inverso del factor que multiplica la aceleración espectral en la ec. 15. Este hecho se tendrá en cuenta al momento de hacer comparaciones entre las diferentes propuestas analizadas.

La norma NEHRP, 1997, propone en el capítulo 6, sección 6.1.3, ec. 6.1.3.-1, que las fuerzas para diseño de elementos no estructurales se calculen de acuerdo con:

$$
F p=\frac{0.4 a_{p} S_{D S} W_{p}}{\frac{R_{p}}{I_{p}}}\left(1+2 \frac{z}{h}\right)
$$

donde:

$S_{D S}:$ es la ordenada del espectro de seudoaceleraciones correspondiente a periodos cortos. 


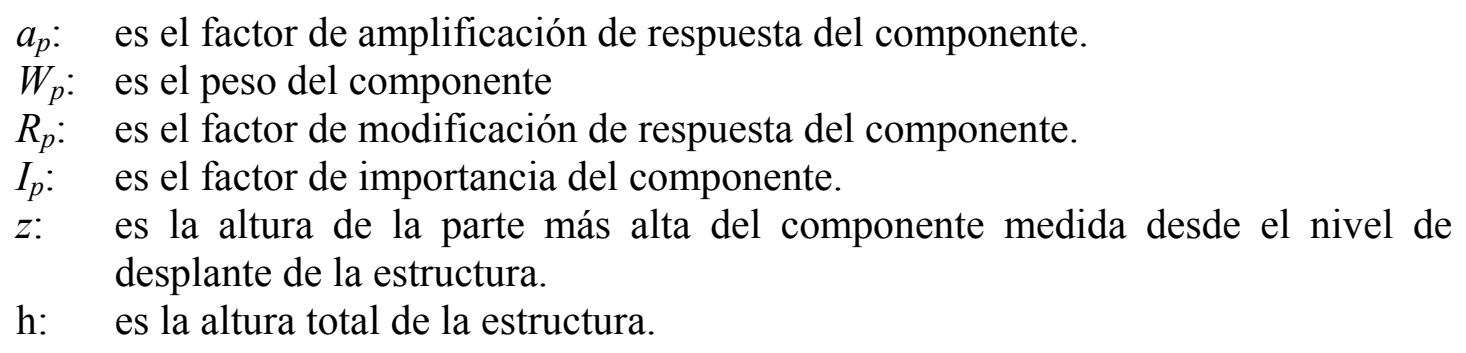

Si se considera para efectos de comparación que se trata de un componente infinitamente rígido, es decir, que $a_{p}=1.0$, que además los factores de modificación de respuesta, $R_{p}, \mathrm{y}$ de importancia, $I_{p}$, son iguales a la unidad, y finalmente, que la meseta del espectro de seudoaceleraciones para periodos cortos es 2.5 veces la aceleración máxima a nivel del terreno, de tal manera que la aceleración para diseño del componente cuando este está a nivel del terreno es igual a la aceleración máxima en la superficie del suelo, resulta, en términos de las variables utilizadas en las otras expresiones analizadas, la siguiente expresión simplificada de la aceleración absoluta en función de la altura sobre la estructura:

$a_{i \max }^{j}=a_{\max }\left(1+2 \frac{y}{H}\right)$

que considera que la aceleración absoluta para diseño de elementos no estructurales tiene una tendencia lineal con la altura, pasando por la aceleración máxima en la superficie del suelo para altura nula, e igual a tres veces la aceleración máxima del terreno para componentes ubicados en la azotea del edificio.

Finalmente, la propuesta de las Normas Técnicas Complementarias para Diseño por Sismo en la ciudad de México de 2001 (NTCS, 2001), propone en el capítulo 8, análisis estático, en la sección 8.1, apéndices y diafragmas, ec. 8.7, que las aceleraciones para diseño sísmico de apéndices son las que le corresponderían si se apoyara directamente en el terreno, multiplicadas por:

$$
1+\frac{c^{\prime}}{a_{0}}
$$

donde:

$a_{0}$ : aceleración máxima en la superficie del terreno.

$c^{\prime}$ : factor que multiplica los pesos a la altura de sujeción del apéndice cuando se evalúan las fuerzas laterales sobre la estructura.

Según la ec. 8.1 de estas normas, este último factor resulta igual a:

$$
c^{\prime}=\frac{c}{Q^{\prime}} h_{i} \frac{\sum W_{i}}{\sum W_{i} h_{i}}
$$


donde:
$c: \quad$ coeficiente sísmico.
$Q^{\prime}:$ Factor de reducción de las fuerzas sísmicas con fines de diseño, función del periodo natural.
$h_{i}: \quad$ altura de la i-ésima masa sobre el desplante.
$W_{i}$ : peso de la i-ésima masa.

$\mathrm{Si}$, para efectos de comparación se supone que el factor de reducción de fuerzas sísmicas, $Q$ ', es igual a 1.0, que la aceleración a la que está sometido el apéndice cuando está apoyado directamente sobre el terreno es igual a la aceleración máxima en la superficie del suelo, $a_{0}$, lo que significaría que se trata de un apéndice infinitamente rígido, y además, que se trata de una edificación con masa uniformemente distribuida en la altura, entonces, la aceleración absoluta en términos de las variables utilizadas en las otras expresiones analizadas y en función de la altura sobre la estructura resulta igual a:

$a_{i \max }^{j}=a_{\max }+2 S a \frac{y}{H}$

De la ec. 20 es claro que la forma modal que se supone para cálculo de fuerzas sísmicas en los diferentes niveles es proporcional a la altura desde el nivel de desplante. De acuerdo con esto y considerando la ec. 14, que permite calcular la altura equivalente asociada a formas modales como las propuestas e igual en este caso a $2 H / 3$, resulta que la aceleración máxima de acuerdo con la ec. 21 a nivel de la altura equivalente es igual a:

$\left.a_{i \max }^{j}\right|_{H_{e q}}=a_{\max }+\frac{4}{3} S a$

El factor 4/3 que multiplica a $S a$ en la expresión anterior, al igual que en el caso de la Norma NSR-98, es producto de la sobrestimación de la cortante basal para un modo de vibración. Este hecho se tendrá en cuenta al momento de hacer comparaciones entre las diferentes propuestas analizadas.

\section{PROPUESTA}

En lo que sigue se considera que la acción sísmica está definida a través del espectro de respuesta de seudoaceleraciones, lo que significa que para un modo de vibración cualquiera, definido a través de su forma modal, $\phi^{j}$, y su frecuencia circular de vibración, $w^{j}$, se conoce la aceleración espectral $S a\left(w^{j}\right)$, su factor de participación, $F P^{j}$, y el factor $\varphi_{i}^{j}$ (ecs. 4, 5 y 6).

Como se mencionó en un párrafo anterior, en este caso se conoce $a_{\max }$, que es igual al valor máximo de $a_{i}^{j}(t)$ cuando $\varphi_{i}^{j}$ es igual a 0.0 y se conoce el valor máximo de $a_{i}^{j}(t)$ cuando $\varphi_{i}^{j}$ es igual a 1.0, que es igual a $S a\left(w^{j}\right)$ (ec. 5). 
A continuación se propone una aproximación basada en el supuesto de que la señal de excitación es armónica de amplitud $A_{1}$. En este caso, la respuesta es otro armónico de la misma frecuencia pero de amplitud diferente, $A_{2}$, y con un ángulo de fase, $\theta$, respecto a la señal de excitación. Finalmente, la suma de estas dos señales es otro armónico de la misma frecuencia y con amplitud, $A_{s}$, dada por la ec. 23:

$$
A_{s}=\sqrt{A_{1}^{2}+A_{2}^{2}+2 A_{1} A_{2} \operatorname{Cos} \theta}
$$

Esta expresión es semejante a la expresión usada para la combinación de respuestas modales, combinación cuadrática completa (CQC por sus siglas en inglés), (Wilson et al., 1981) que permite estimar la esperanza del valor máximo de una serie de tiempo que resulta de la suma de otras series de tiempo sobre las que se conocen sus valores máximos y propiedades de los sistemas de un grado de libertad; frecuencias de vibración, amortiguamientos y factores de participación, $F P$, por las cuales se filtró una única señal de entrada.

En el caso que nos ocupa y de acuerdo con la ec. 23, la esperanza de la aceleración absoluta máxima del grado de libertad $i$ debida al modo $j, a_{i \max }^{j}$, se puede expresar en términos de la aceleración máxima del suelo, $a_{\max }$, el factor $\varphi_{i}^{j}$ y la aceleración relativa máxima del sistema, $z_{\max }^{j}$, como:

$a_{i \max }^{j}=\sqrt{a_{\max }^{2}+\left(\varphi_{i}^{j} \ddot{z}_{\max }^{j}\right)^{2}+2 \rho a_{\max }\left(\varphi_{i}^{j} \ddot{z}_{\max }^{j}\right)}$

donde $\rho$ es un factor que varía entre -1 y 1 y que mide la correlación entre las dos señales que se están sumando: $a_{\max } \mathrm{y}\left(\varphi_{i}^{j} z_{\max }^{j}\right)$. Si $\rho=0$, significa que las dos señales no están correlacionadas y entonces el método converge al conocido método de Rosenblueth (Newmark y Rosenblueth, 1971), o de la raíz cuadrada de la suma de los cuadrados (SRSS por sus siglas en inglés).

El método CQC como está propuesto en la literatura, para la combinación de dos o más señales provenientes de una sola señal de entrada y filtradas por diferentes sistemas de un solo grado de libertad, expresa el factor de correlación, $\rho$, en función de la relación entre las frecuencias naturales de vibración y los amortiguamientos de los sistemas de un grado de libertad. Además, el factor de correlación, $\rho$, varía entre 0.0 y 1.0 y el signo del término cruzado está dado por los signos de los factores de participación $F P^{j}$.

En la propuesta que se hace, el signo del doble producto de la ec. 24 está dado por el producto del signo del factor de correlación de acuerdo con las consideraciones que se relacionan a continuación y por el signo del factor $\varphi_{i}^{j}$.

Es claro que la aceleración absoluta máxima, $a_{i \max }^{j}$, en un sistema de un grado de libertad en el que $\varphi_{i}^{j}=1.0$ es igual a cero para periodos de vibración muy altos, lo que significa, de 
acuerdo con la ec. 24, que el factor de correlación, $\rho$, debe ser igual a -1.0 y que la aceleración relativa $z^{j}{ }_{\max }$ es igual a $a_{\max }$.

Estas afirmaciones se pueden confirmar si se hace referencia a la función de transferencia, $F T$, entre la aceleración en la base y la aceleración relativa de un sistema de un grado de libertad,

$$
F T=\frac{\beta^{2}}{\left(1-\beta^{2}\right)+(2 \xi \beta) i}
$$

En ésta, la amplitud tiende a 1.0 para una relación del periodo del sistema al periodo de la excitación, $\beta$, tendiendo a $\infty$, y para esta misma relación de periodos el ángulo de fase tiende a $180^{\circ}$, es decir, que las dos señales, de entrada y de salida, son iguales pero de signos contrarios.

De la misma función de transferencia se concluye que para el estado de resonancia; período de la excitación igual al período de la estructura, $\beta=1.0$, el ángulo de fase es igual a $90^{\circ} 10$ que significa que la correlación entre las señales de entrada y salida es igual a cero: $\rho=0.0$.

En el otro extremo, para un sistema infinitamente rígido, $\beta=0.0$, el ángulo de fase es igual a $0^{\circ}$ y la amplitud de la función de transferencia es igual a 0.0 , lo que significa que la correlación es perfecta, $\rho=1.0$, y que la aceleración relativa $z^{j}{ }_{\max }$ es igual a 0.0 , es decir, que la aceleración absoluta, $a_{i \max }^{j}$, es igual a $a_{\max }$.

Con base en estas consideraciones, se propone que el factor de correlación, $\rho$, varíe con el periodo de la estructura, $T$, relativo al periodo en el que el espectro de respuesta de aceleraciones es máximo e igual a $S a_{\max }, T_{s}$, de acuerdo con las siguientes expresiones:

$$
\begin{array}{ll}
\rho=\left(\frac{S a_{\text {max }}-S a(T)}{S a_{\text {max }}-a_{\text {max }}}\right)^{2} & 0.0<T<T_{S} \\
\rho=-\sqrt{\frac{S a_{\text {max }}-S a(T)}{S a_{\text {max }}}} & T>T_{S}
\end{array}
$$

En estas, $\rho=1.0$ para $T=0.0, \rho=0.0$ para $T=T_{s}, \mathrm{y} \rho=-1.0$ para $T \rightarrow \infty$.

La variación del coeficiente de correlación con el periodo en los dos intervalos definidos está determinada por el mejor ajuste de la expresión propuesta a soluciones exactas usando series de tiempo. Igualmente, se considera que no es la relación de periodos, $\beta$, la que define el coeficiente de correlación, como en el caso de señales armónicas, sino que puede ser más bien las relaciones entre las amplitudes de los espectros de respuesta las que definen estas correlaciones. 
Finalmente, el valor de $z_{\text {max }}^{j}$, la aceleración relativa máxima, se estima sabiendo que la aceleración absoluta máxima, $a_{i \max }^{j}$, en el caso en el que $\varphi_{i}^{j}=1.0$, es igual a la aceleración espectral, $S a(T)$. Reemplazando estos valores en la ec. 24 , se tiene:

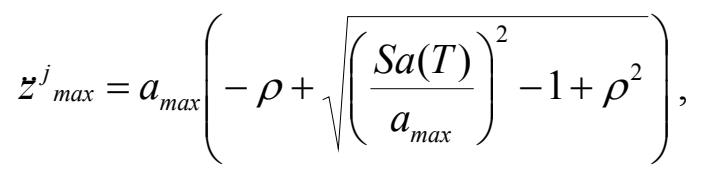

que junto con las ecs. 24, 26 y 27, define completamente el problema de calcular $a_{i \max }^{j}$ en función de $a_{\max }, S a(T), T_{s}, S a_{\max } \mathrm{y} \varphi_{i}^{j}$, este último definido por las ecs. 6 y 4.

Las expresiones propuestas satisfacen las condiciones límite señaladas en párrafos anteriores: para periodos de vibración altos en los que $S a(T)$ tiende a 0.0 , el coeficiente de correlación, $\rho$, tiende a -1.0 y la aceleración relativa $z^{j}{ }_{\max }$ tiende a $a_{\max }$. Igualmente, para periodos muy bajos, en los que $S a(T)$ tiende a $a_{\max }$, el coeficiente de correlación tiende a 1.0 y la aceleración relativa $z^{j}{ }_{\max }$ tiende a 0.0 .

Las ecs. 26 y 27 tienen algunas limitaciones que se señalan a continuación. En primer lugar, la ec. 26, que permite calcular el coeficiente de correlación en el intervalo de periodos $0.0<T<T_{s}$, resulta en un valor mayor que $1.0 \mathrm{si} S a(T)<a_{\max }$. En este caso, se propone considerar $S a(T)=a_{\max }$, lo que resulta en $\rho=1.0, z^{j}{ }_{\text {max }}=0.0 \mathrm{y} a_{i \max }^{j}=a_{\max }$, es decir, el mismo resultado que se obtiene para $\mathrm{T}=0.0$.

En segundo lugar, la ec. 27 aplicada en el intervalo $T>T_{s}$, puede resultar en valores imaginarios en la raiz cuadrada de la ec. 28 para valores muy bajos de $S a(T)$;

$$
\frac{S a}{a_{\max }}<\frac{a_{\max }}{S a_{\max }}
$$

En este caso, se propone considerar $\rho=-1.0$ y continuar el cálculo de los demás valores con las expresiones propuestas.

\section{RESULTADOS}

A continuación se comparan los resultados exactos usando para su cálculo la respuesta en el dominio del tiempo de sismos registrados, con los resultados de la propuesta presentada. Igualmente, se comparan los resultados exactos y la propuesta que se hace, con la actual propuesta de la norma NSR-98, con la propuesta de las NTCS-2001, ambas corregidas para evitar el factor de sobreestimación en la cortante basal que se calcula para un solo modo, con la propuesta de la Norma NEHRP (1997), y con la propuesta de Paulay y Priestley (1992). 
Para lo anterior, se seleccionaron seis acelerogramas con contenidos frecuenciales y formas espectrales muy diferentes.

Tabla 1. Registros usados para cálculo y comparación de resultados

\begin{tabular}{|c|}
\hline ESTACIÓN \\
\hline El Centro, 1940 \\
\hline Tokachi-Oki, \\
\hline México, SCT, 1985 \\
\hline Chile, Viña del Mar, 1985 \\
\hline Loma Prieta, Corralitos, 1989 \\
\hline Loma Prieta, Capitola Fire station, 1989 \\
\hline
\end{tabular}

A continuación se muestran los valores exactos de la aceleración absoluta en función de la altura normalizada de la edificación, $(y / H)$, para un valor igual a $2.0 \mathrm{del}$ exponente $k$ que define la forma modal, para una razón de amortiguamiento igual a 0.03, para diferentes periodos de vibración y para diferentes sismos. En todas las gráficas se incluye la solución exacta, la solución propuesta en este trabajo, la solución propuesta por Paulay y Priestley (1992), y las propuestas de las Normas, NEHRP (1997), NSR-98 y propuesta de NTCS-2001, estas dos últimas corregidas para hacerlas comparables.

De las figs. 1, 2, 3 y 4 es claro como las aceleraciones absolutas calculadas de acuerdo con la propuesta presentada en este trabajo se ajustan con precisión a lo largo de toda la altura de la edificación a las aceleraciones absolutas calculadas de manera exacta. Aunque las propuestas de Paulay y Priestley, y la de la norma NSR-98 siguen las tendencias de los valores exactos, la primera presenta diferencias que alcanzan el $50 \%$ de error principalmente en la máxima altura de la edificación, $y / H=1.0$, y la segunda propuesta presenta estos mismos errores consistentemente a nivel de la superficie del suelo en donde es claro que la aceleración absoluta máxima es igual a la aceleración máxima del suelo y no a la mitad de este valor. Esta última propuesta también presenta diferencias con la solución exacta que alcanzan el $50 \%$ de error principalmente en la máxima altura de la edificación.

La propuesta de las normas NEHRP (1997) es independiente del espectro de seudoaceleraciones, lo que la hace completamente inapropiada para aceleraciones espectrales relativas a la aceleración máxima del suelo menores a 1.5 veces. Igualmente, para el caso de aceleraciones espectrales mayores a 3.5 veces la aceleración máxima del suelo, esta norma proporciona valores de aceleración muy bajos.

La propuesta de las normas NTCS-2001, aunque función de la aceleración espectral, sistemáticamente presenta una sobre estimación a nivel de la altura equivalente de la estructura de aproximadamente una vez la aceleración máxima del suelo, que lleva a sobre estimaciones excesivamente grandes a lo largo de la altura total de la edificación. 


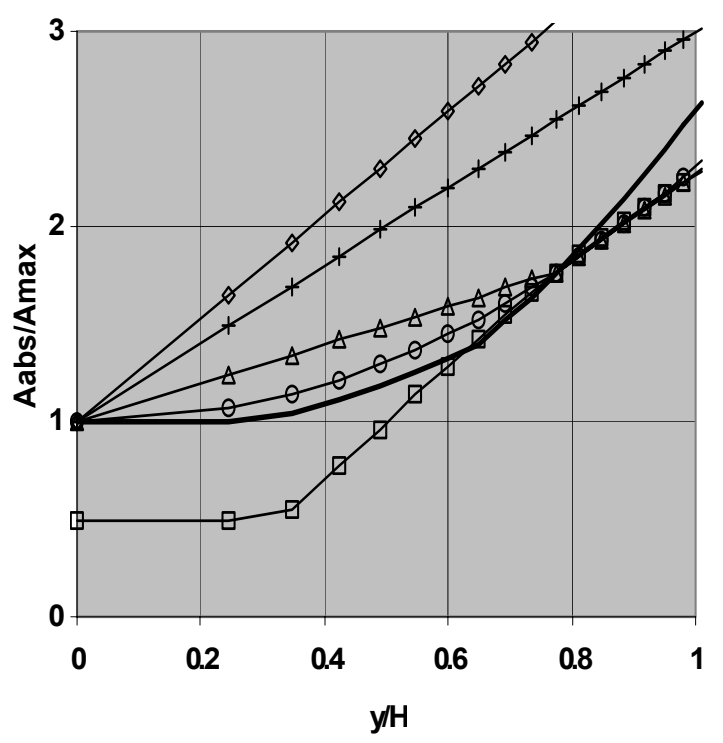

$\mathrm{T}=0.5 \mathrm{~s}$

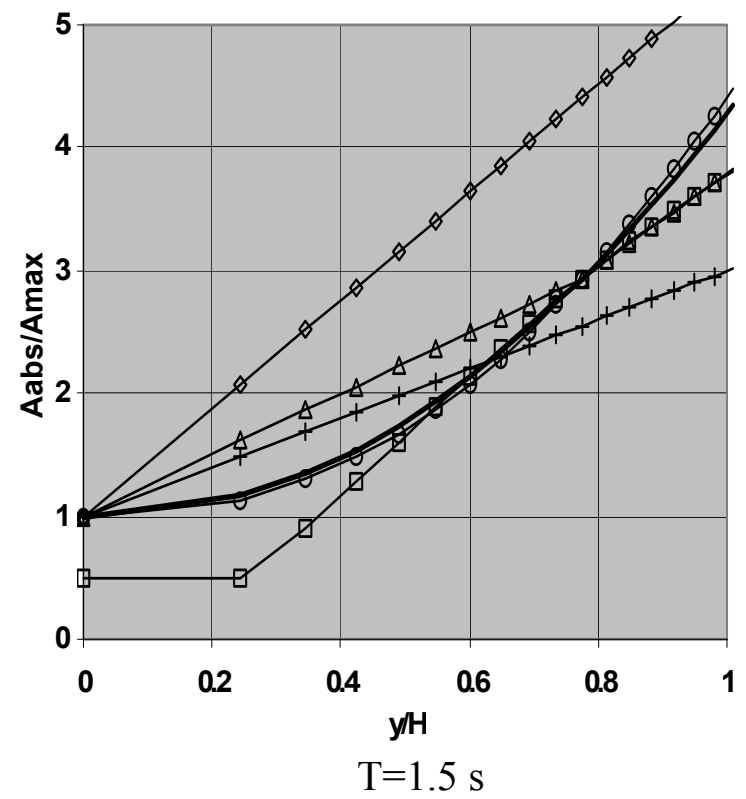

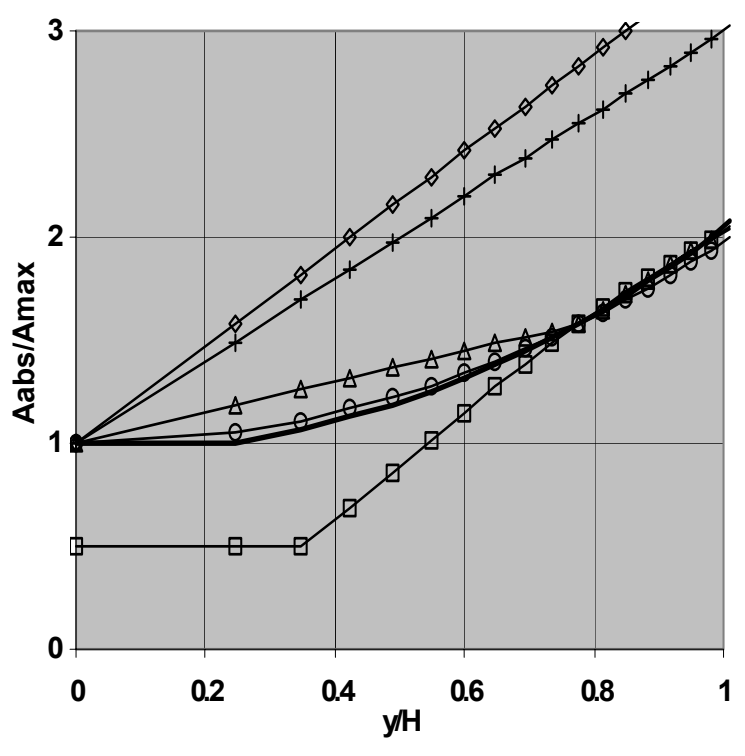

$\mathrm{T}=1.0 \mathrm{~s}$

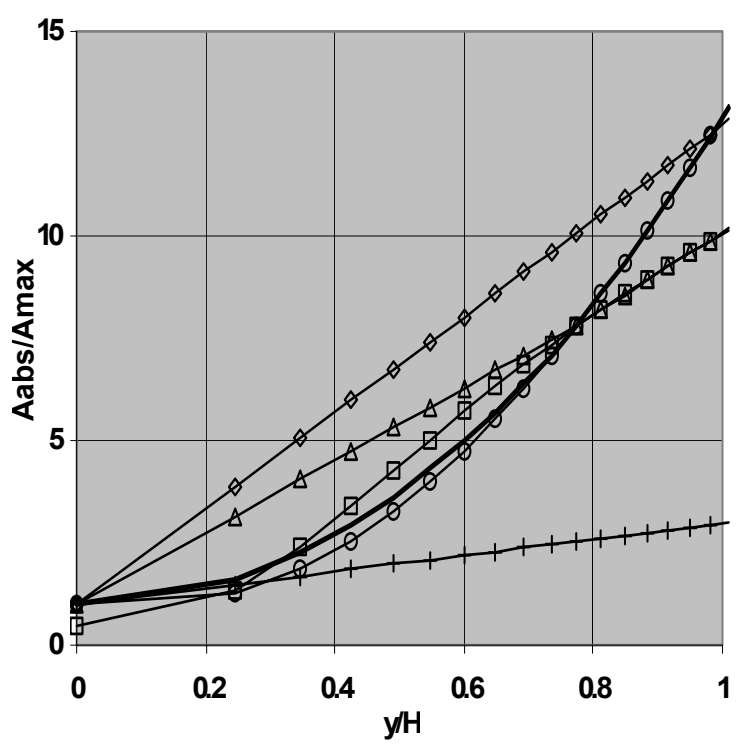

$\mathrm{T}=2.0 \mathrm{~s}$

Exacta $\neg$ Propuesta $\triangle$ Paulay Priestley $\square$ NSR-98 $\multimap$ NTC

— NEHRP

Figura 1. Aceleraciones absolutas normalizadas a la aceleración máxima del suelo en función de la altura normalizada de la estructura, $y / H$, para el sismo de México, 1985. 

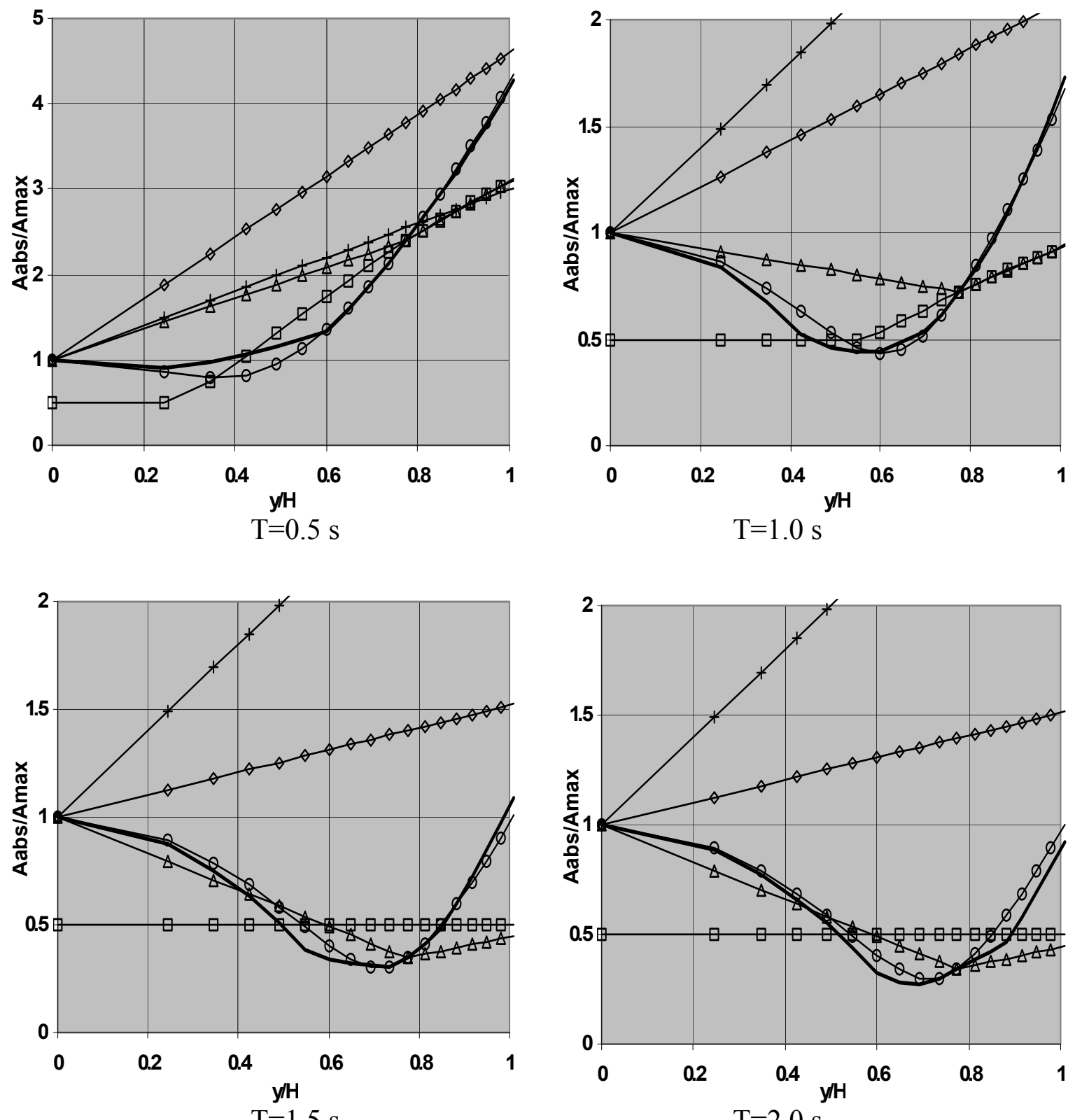

$\mathrm{T}=1.5 \mathrm{~s}$

$\mathrm{T}=2.0 \mathrm{~s}$

Figura 2. Aceleraciones absolutas normalizadas a la aceleración máxima del suelo en función de la altura normalizada de la estructura, $y / H$, para el sismo de Loma Prieta, Corralitos, 1989. 

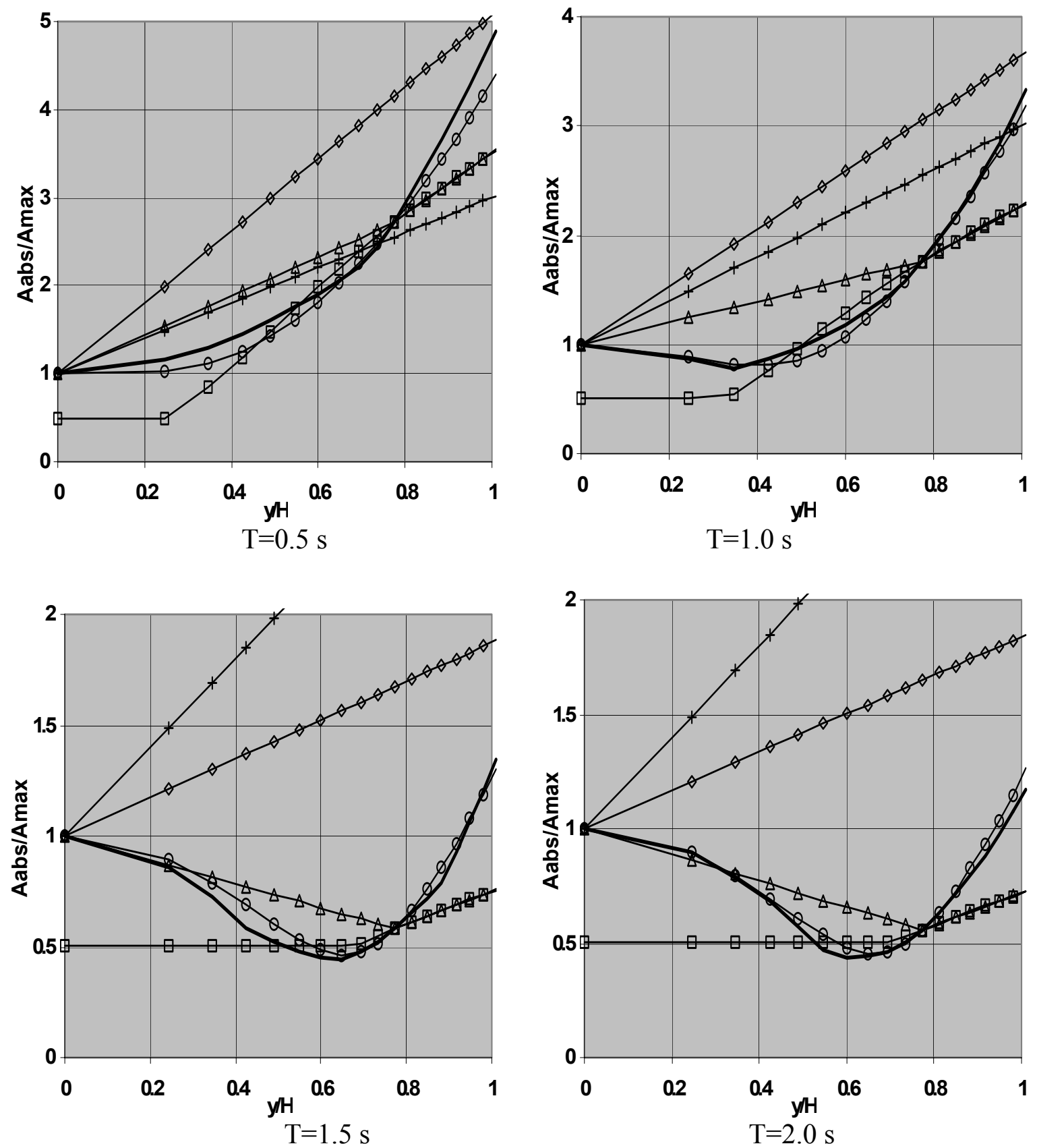

Exacta $\neg$ Propuesta $\triangle$ Paulay Priestley $\square$ NSR-98 $\neg$ NTC $\longrightarrow$ NEHRP

Figura 3. Aceleraciones absolutas normalizadas a la aceleración máxima del suelo en función de la altura normalizada de la estructura, $y / H$, para el sismo de El Centro, 1940. 

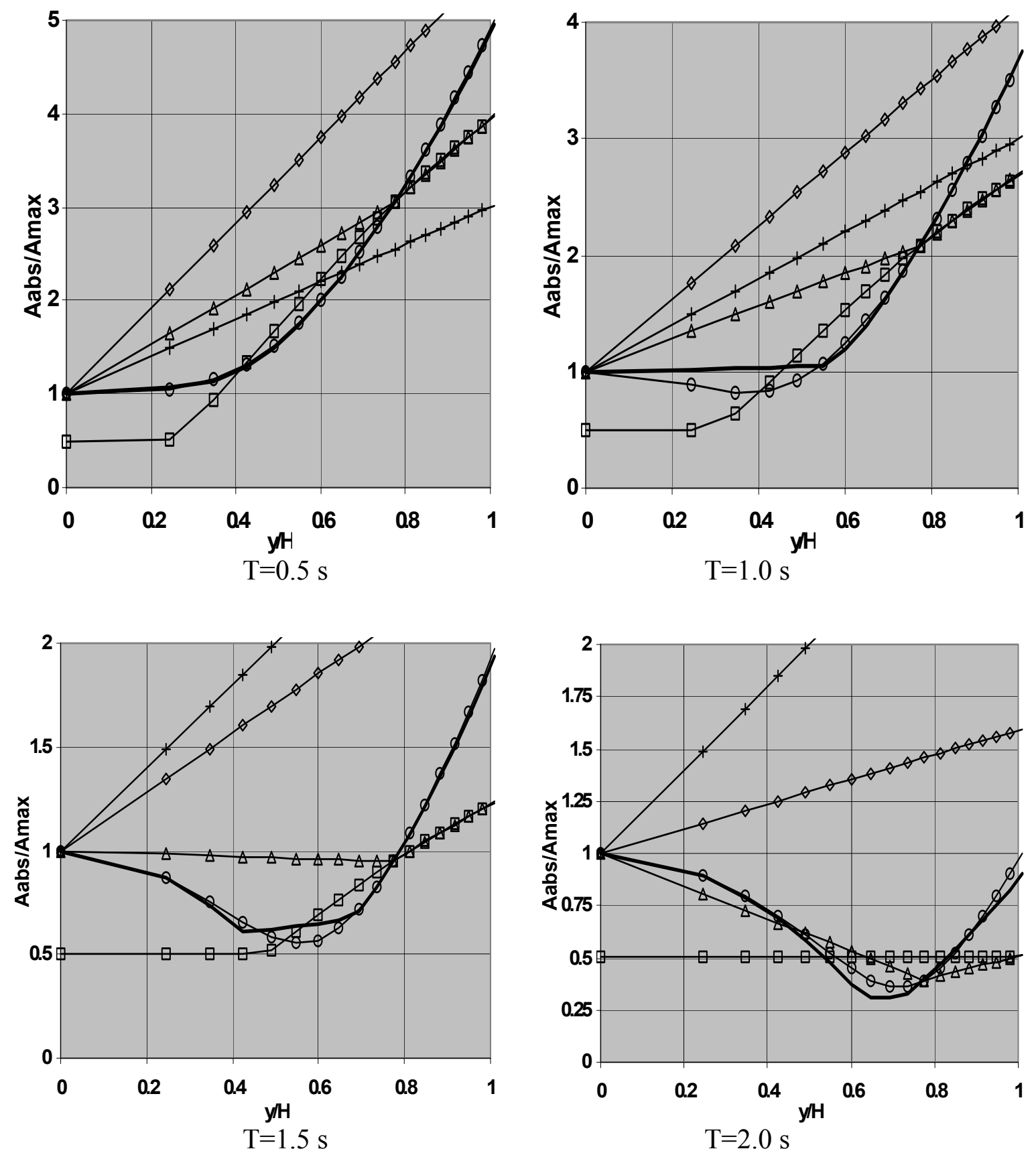

Exacta $\multimap$ Propuesta $\triangle$ Paulay Priestley $\square$ NSR-98 $\multimap$ NTC $\multimap$ NEHRP

Figura 4. Aceleraciones absolutas normalizadas a la aceleración máxima del suelo en función de la altura normalizada de la estructura, $y / H$, para el sismo de Viña del Mar, Chile, 1985. 
Para dar una medida más concreta de la precisión de cada una de las soluciones analizadas se calculó el promedio del porcentaje de error de las propuestas respecto a la solución exacta en diez puntos de la altura de la edificación, para periodos de vibración entre $0.1 \mathrm{~s} \mathrm{y} 2.0 \mathrm{~s}$ cada $0.1 \mathrm{~s}$, para dos razones de amortiguamiento: 0.05 y 0.03 ; para tres valores de $k$, que define la forma modal adoptada; y todo lo anterior para cada uno de los sismos propuestos en la tabla 1. El número de respuestas en las que se basa el cálculo presentado y para cada sismo es 1320. En la tabla 2 se muestran los resultados de los cálculos descritos.

Tabla 2. Promedio del porcentaje de error respecto a la solución exacta

\begin{tabular}{|c|c|c|c|c|c|c|c|c|c|c|c|c|c|c|c|}
\hline \multirow[t]{3}{*}{ SISMOS } & \multicolumn{3}{|c|}{$\begin{array}{c}\text { PROPUESTA } \\
\text { ERROR (\%) }\end{array}$} & \multicolumn{3}{|c|}{$\begin{array}{c}\text { PAULAY- } \\
\text { PRIESTLEY } \\
\text { ERROR(\%) } \\
\end{array}$} & \multicolumn{3}{|c|}{$\begin{array}{c}\text { NSR98 } \\
\text { ERROR(\%) }\end{array}$} & \multicolumn{3}{|c|}{$\begin{array}{c}\text { NTC, 2001 } \\
\text { ERROR(\%) }\end{array}$} & \multicolumn{3}{|c|}{$\begin{array}{l}\text { NEHRP, } 1997 \\
\text { ERROR(\%) }\end{array}$} \\
\hline & \multicolumn{3}{|c|}{$\bar{K}$} & \multicolumn{3}{|c|}{$\bar{K}$} & \multicolumn{3}{|c|}{$\mathbf{K}$} & \multicolumn{3}{|c|}{$\bar{K}$} & \multicolumn{3}{|c|}{$\bar{K}$} \\
\hline & 1.0 & 1.5 & 2.0 & 1.0 & 1.5 & 2.0 & 1.0 & 1.5 & 2.0 & 1.0 & 1.5 & 2.0 & 1.0 & 1.5 & 2.0 \\
\hline MEXICO & 3.4 & 3.4 & 3.4 & 7.7 & 8.8 & 11.8 & 17.5 & 16.0 & 15.3 & 45.1 & 57.1 & 66.8 & 41.1 & 46.7 & 51.9 \\
\hline CENTRO & 6.0 & 6.0 & 6.0 & 18.5 & 22.2 & 24.5 & 16.7 & 17.5 & 18.4 & 78.5 & 90.3 & 99.5 & 106.5 & 121.2 & 132.6 \\
\hline CORRAL & 9.2 & 9.2 & 9.2 & 27.3 & 27.8 & 28.7 & 27.5 & 28.2 & 28.9 & 110.0 & 120.7 & 128.9 & 119.0 & 121.0 & 122.5 \\
\hline LP-CAP & 9.4 & 9.4 & 9.4 & 16.0 & 20.8 & 24.3 & 18.2 & 19.1 & 20.0 & 64.3 & 76.8 & 86.6 & 81.3 & 92.9 & 102.1 \\
\hline $\begin{array}{l}\text { VIÑA } \\
\end{array}$ & 5.9 & 5.9 & 5.9 & 18.4 & 22.6 & 25.7 & 17.5 & 18.7 & 19.8 & 71.2 & 83.7 & 93.5 & 88.3 & 101.6 & 111.9 \\
\hline MITOKI & 7.0 & 7.0 & 7.0 & 11.1 & 17.4 & 21.7 & 17.1 & 17.5 & 18.1 & 47.6 & 60.9 & 71.3 & 50.3 & 57.5 & 64.1 \\
\hline
\end{tabular}

Es claro de la tabla 2 y de las figs. 1, 2, 3 y 4 que, en términos de la desviación respecto a la solución exacta, la propuesta de este trabajo presenta errores hasta tres veces menores que la propuesta de Paulay Priestley o la propuesta de la norma NSR98. Respecto a las propuestas de las normas NEHRP, 1997 o la propuesta de normas NTC, 2001, la propuesta presentada es considerablemente más ajustada a la solución exacta. Por otro lado, es claro de las figs. 1, 2 ,3 y 4 que los errores de la propuesta presentada se mantienen dentro de los mismos niveles en toda la altura de la edificación, mientras que en las otras propuestas analizadas el error varía con la altura desde valores muy bajos en los puntos de control, a valores que superan el 100\% para determinadas alturas.

Para efectos reglamentarios prácticos cabe la pregunta de cómo considerar modos superiores al fundamental. A falta de un procedimiento más riguroso, sobre el que se está trabajando actualmente, que considere la estimación de aceleraciones absolutas debidas a la combinación de $n$ modos, se propone utilizar la misma estrategia que se usa en los métodos de la fuerza horizontal equivalente, que están basados en una sola forma modal, y que por ejemplo, para el caso de estimación de la cortante basal, considera toda la masa de la edificación y no solo la masa asociada a ese modo, lo que se puede interpretar como un factor de amplificación que se aplica a todas las respuestas calculadas rigurosamente para ese solo modo.

El factor $(k+1)^{2} /(2 k+1)$, donde $k$ es el exponente que define la forma modal en consideración $\left(y^{k}\right)$, y que se muestra en las ecs. 15 y 16, es el factor multiplicador que resultaría del razonamiento anterior y que se propone afecte a las aceleraciones absolutas calculadas con el procedimiento propuesto para una sola forma modal con el propósito de considerar los incrementos de aceleración por la participación de otros modos en la respuesta. 
Para efectos reglamentarios igualmente válida es la pregunta de qué pasa con las aceleraciones absolutas cuando la estructura no se comporta elásticamente. Independientemente de si el comportamiento de la estructura es o no elástico, la aceleración máxima absoluta a nivel del suelo sigue siendo $a_{\max }$. Ahora, ¿Qué pasa con la aceleración máxima absoluta de un sistema de un grado de libertad si idealizamos su comportamiento como elastoplástico perfecto, como es usual para definir tendencias y comportamientos? Del equilibrio del sistema en el instante en que se alcanza el desplazamiento máximo se deduce que la aceleración absoluta es igual a la fuerza resistente del sistema dividida por su masa, y que esta aceleración puede ser muy cercana a la máxima absoluta del sistema debido a que en otros instantes se encuentra presente la fuerza de amortiguamiento.

Todo lo anterior puesto en el contexto del método propuesto y suponiendo que la estructura plastificada se deforma de manera semejante a la forma modal fundamental que resulta de un análisis elástico de la estructura, se propone que para considerar casos en los que ésta ingresa al intervalo plástico basta con considerar el espectro de respuesta inelástico asociado a la ductilidad seleccionada, a cambio del espectro de respuesta elástica. Aquí cabe señalar que para periodo nulo, los espectros elástico e inelástico, independientemente de la ductilidad a la que esté asociado este último, indican la misma $a_{\max }$, hecho que ya fue señalado en el párrafo anterior.

\section{CONCLUSIONES}

Se presenta una propuesta para la estimación de la aceleración absoluta máxima en los diferentes niveles de un sistema de múltiples grados de libertad en función de la forma modal y del espectro de respuesta de aceleraciones, que tradicionalmente se propone en los reglamentos. Esta propuesta tiene un error promedio cuando se compara con la solución exacta calculada usando series de tiempo de aproximadamente $7 \%$ que contrasta con errores promedio del orden de $20 \%$, $60 \%$ y $80 \%$ en otras propuestas analizadas.

La propuesta está basada en la combinación de la respuesta modal con la señal de excitación cuando estas se consideran armónicas. En términos generales, las expresiones propuestas y los cálculos involucrados no difieren mucho de los comúnmente utilizados para la combinación de respuestas modales, como en el conocido método CQC.

La propuesta puede ser incluida formalmente en los reglamentos de diseño sismo resistente para estimar las aceleraciones máximas a las que estarían sometidos elementos no estructurales o apéndices ubicados a diferentes alturas que pudieran considerarse como infinitamente rígidos.

\section{REFERENCIAS}

AIS (1999), "Normas colombianas de diseño y construcción sismo-resistente, Ley 400 de 1997, decreto 33 de 1998”, Asociación Colombiana de Ingeniería Sísmica (AIS), Santafé de Bogotá.

Clough, R W y J Penzien (1993), Dynamics of structures, segunda edición, McGraw-Hill. 
Igusa, T y A Der Kiureghian (1985), "Generation of floor response spectra including oscillatorstructure interaction”, Earthquake Engineering and Structural Dynamics, Vol. 13, pp. 661-676.

NEHRP (1997), "Recommended provisions for seismic regulations for new buildings and other structures", 1997 edition.

Newmark, N M y E Rosenblueth (1971), Fundamentals of Earthquake Engineering, PrenticeHall.

Paulay, T y M J N Priestley (1992), Seismic design of reinforced concrete and masonry buildings, John Wiley \& Sons.

NTCS-2001 (2001), "Propuesta de normas técnicas complementarias para diseño por sismo", http://www.smie.org.mx/articulos , abril.

Singh, M P (1980), "Seismic design input for secondary systems", ASCE Journal of Structural Engineering, Vol. 106, pp. 505-517.

Singh, M P y A M Sharma (1985), "Seismic floor spectra by mode acceleration approach", ASCE Journal of Engineering Mechanics, Vol. 111, No.11.

Wilson, E L, A Der Kiureghian y E R Bayo (1981), “A Replacement for the SRSS Method in Seismic Analysis”, Earthquake Engineering and Structural Dynamics, Vol. 9, pp. 187-192. 